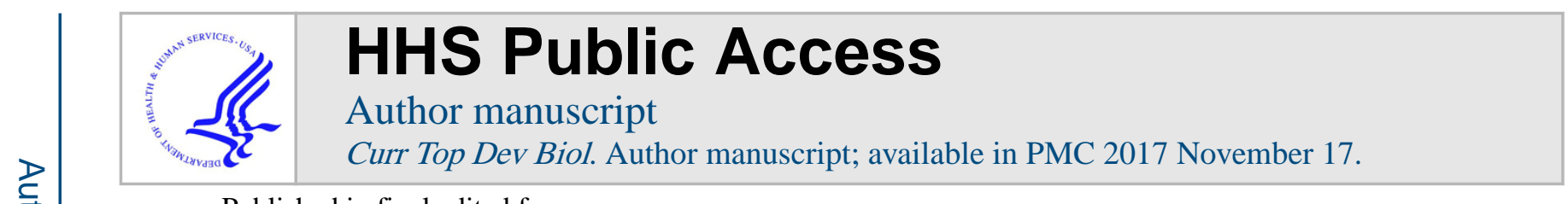

Published in final edited form as:

Curr Top Dev Biol. 2016 ; 116: 315-330. doi:10.1016/bs.ctdb.2015.11.001.

\title{
CONNECTOMICS, THE FINAL FRONTIER
}

\author{
Scott W. Emmons \\ Department of Genetics, Dominick P. Purpura Department of Neuroscience, Albert Einstein \\ College of Medicine
}

It may seem foolish to use the word "final," like the word "never," to describe anything in science. Nevertheless, from today's perspective, what the connections are in our nervous system, the connectome, seems to be the last major gap in our knowledge of the phenotype, certainly in that aspect of the phenotype which is neuroanatomy. How mind emerges from brain remains one of the great mysteries. Through over a century of neuroscience research we know that thought, consciousness, and behavior emerge from electrical circuits in the brain. As long as the structure of these circuits remain undescribed, knowledge of the properties of the individual cellular components - the neurons, glia, and muscles — and the nature of their pairwise connections taken one-by-one will be insufficient to understand the function of the complex system they create when taken all together. The information required to build this structure lies somewhere in our genes, but we don't know where.

This conceptually elementary aspect of the phenotype remains unknown today because tools are still not available to access it. The essential tool that's required, the electron microscope (EM), is cumbersome and has a tiny viewing window. It is unmatched to the size of the nervous systems of even most small invertebrates. Sydney Brenner, recognizing this, looked for the smallest animal he could find with a nervous system that might be reachable and as is well-known, settled on the free-living nematode worm Caenorhabditis elegans (Brenner, 2009; Emmons, 2015). After 15 years of effort, in 1986 the connectivity of each of the 118 classes of neurons in the 302-neuron nervous system of the adult of one sex, the hermaphrodite (the "female" sex of $C$. elegans), was published - the first connectome (White et al., 1986). But this was the limit. Even the nervous system of the male worm could not be fully deciphered and no similar work was attempted for over a decade (Sulston et al., 1980). With the male, size was not the issue. The problem was in the accounting. The male nervous system is larger than that of the hermaphrodite and there were too many connections between too many branched neurons in the posterior ganglia that control mating to be managed by the pencil-and-paper methods of the 1970's (D. Albertson, personal communication). While EM imaging and methods to decipher electron micrographs still struggle today to gain traction on larger nervous systems, for the $C$. elegans male the PC was sufficient to overcome the accounting problem and the mating circuits were described in 2012 (Jarrell et al., 2012).

\section{How does it work? How is it built?}

As Brenner pointed out in his first major paper on $C$. elegans, for the nervous system there are two chief and somewhat independent questions: how does it work and how is it built (Brenner, 1974)? It was hoped that knowledge of structure would shed light on both of these 
questions and this expectation has not met with disappointment. An early experimental study guided by the structure revealed how the worm moves forward and backward and responds to light touch (Chalfie et al., 1985). Since that time a great number of experimental analyses have uncovered the functions of individual neurons and elucidated circuits driving further components of the worm's behavior (see WormBook.org for reviews). In the case of the male, the structure of the mating circuits gave insight into the complex decision-making process by which the male integrates inputs from a large number of sensory neurons to guide its efforts to inseminate the hermaphrodite (Jarrell et al., 2012).

Similarly for how it is built — and, crucially, how it is genetically specified — knowledge of the structure made it possible to contemplate what cellular and molecular mechanisms might underlie its formation. John White considered the issue carefully (White, 1985; White et al., 1983; White et al., 1986). His reconstruction revealed that the nervous system of the hermaphrodite has a rather simplified structure compared to those of other animals. The component neurons, constant and stereotyped in number and identity, are largely unbranched and run for long distances in bundles with a constant and small set of neighbors (Figure 1). The neighbors are the same in every animal. This describes the ventral nerve cord and even the more complex nerve ring, which is essentially a large parallel bundle of fibers running around the pharynx. As a neuron can only establish a synaptic connection to an adjacent neuron, and moreover, since in $C$. elegans it does so with a large fraction of these (average of $45 \%$ for four examples examined), the neural cell adhesion proteins that presumptively establish and stabilize these bundles must constitute one set of important genetic determinants of overall connectivity.

In order to adhere to another neuron, a given neuron must first come into contact with it. As pointed out by White, in $C$. elegans opportunities for contact are determined initially by the cell lineage. Only a few cells undertake long-range migrations to relocate from their place of birth. Thus, the neighbors available for association during outgrowth of a neurite are the cells born nearby. In the lineage, which is reproducible from animal to animal, cells of a given type do not in general arise together from specialized branches (the gut, a clone, is the exception that proves this rule). Rather, the distribution of cells across the lineage appears somewhat chaotic with disparate cell types related as siblings or cousins (Sulston, 1983; Sulston et al., 1983). The underlying principle seems to be that cells are generated near or at the location where they are needed. In the case of neurons, they are born near the neurons they initially fasciculate with. Probably the usually single outgrowing growth cone is drawn from its starting point into the neighborhood that maximizes its set of adhesive interactions. As a result of this mechanism, in addition to the cell recognition and adhesion genes that establish and stabilize process bundles, another set of genes that contribute to specification of nervous system architecture is apparently the set of developmental regulatory genes that underlie determination of cell fate in the lineage.

Cell contact alone is not sufficient to specify the connectome. Although a neuron synapses with a significant fraction of its neighbors, it does not synapse with all of them. Since the number of choices is small, White suggested a second signal might simply be neurotransmitter/receptor matching. A simple model would be that for each pair of neurons or neurons and muscles, cell contact triggers a recognition event that results in synapse 
formation with a certain, genetically-specified probability depending on the cells involved. Closer analysis reveals this model is too simple. To address the question, Richard Durbin examined the cell contacts and synapses made in a single animal by presumptively equivalent neurons, the members of homologous left/right pairs (Durbin, 1987). Such pairs exhibit a natural variability in the extent of their contacts with neighbors as well as in the number of synaptic connections they make with them. If synapse formation occurred with a certain fixed probability dependent upon the extent of contact, then the amount of contact with a particular target and the number of synapses made to it should co-vary. But Durbin found that they did not: the member of a pair that made more contact with a particular partner did not necessarily make more synapses with it. A similar result has been found when synapse size as well as synapse number is taken into account (C. Brittin and S. W. Emmons, unpublished observations).

A number of experimental studies in $C$. elegans have revealed something about additional contact-independent mechanisms. First, formation of a presynaptic structure can be regulated - induced or inhibited — by signals from cells not directly involved in the synapse itself. The HSN motor neuron is induced to form a pre-synaptic structure intended for muscle by a signal from an epithelial cell, and this occurs at a particular location on the HSN neuron whether the muscle target is present or not (Shen and Bargmann, 2003). The AIY interneuron is similarly induced to form presynaptic structures by a signal from a glial cell, while the DA09 motor neuron is prevented from forming pre-synaptic structures in dendritic regions by signals from epithelial cells (Colón-Ramos et al., 2007; Klassen and Shen, 2007; Poon et al., 2008; Shao et al., 2013).

Second, synapse formation at one location on a neurite can influence the formation of a synapse to a different target elsewhere on the same neurite. In the case of the HSN neuron, if formation of a pre-synaptic structure at the proper location is not induced, pre-synapses at inappropriate locations adjacent to inappropriate targets are not pruned away. Pruning requires the protein degradation pathway whose activity is spatially regulated by the synapse-inducing signal (Ding et al., 2007). In the case of a class of body wall inhibitory motor neurons, induction of pre-synaptic sites on the ventral side of the body is necessary to prevent their formation in dendrites on the dorsal side (Howell et al., 2015). It seems there is a balance wherein the multiple synapses formed by a neuron are in competition with one another.

The picture that emerges is one in which neurons are somewhat promiscuous for synapse formation. These synaptogenic tendencies are directed towards the small set of potential partners in contact. Assembly of a pre-synaptic structure is governed by both internal mechanisms and external signals, likely through regulation of a dynamic balance between biosynthetic and break down pathways or possibly transport pathways within neurites. For neurons that make en passant synapses, where to assemble a presynaptic density to communicate with a partner that may be in contact over an extended region is potentially a structural problem that might be solved by an external signal. This is not an issue for $C$. elegans alone. A recent EM reconstruction of a region of mouse neocortex has shown that here too neurites make en passant synaptic connections to their targets (Kasthuri et al.). 


\section{The C. elegans male mating circuits - a more typical neuropil}

All of these features - a small, stereotyped number of unbranched neurons in a highly structured arrangement, constant from animal to animal, forming en passant synapses guided by outside signals - might seem to make the $C$. elegans hermaphrodite nervous system a poor representative of most animal nervous systems for the purpose of figuring out how synaptic connections are in general specified. Certainly the structure does not resemble the textbook image in which a neuron sends out an axon behind a growth cone that navigates long distances following pathfinding guidance cues until it reaches its target, which it recognizes and where it forms a synapse.

Such is the typical depiction of a neuron that has guided thinking about the problem of synaptic specificity for many years. In 1963, after he observed in the fish visual system that outgrowing axons bypassed potential target neurons until they arrived at the location of their correct partners, Roger Sperry pointed out that some kind of genetically-specified molecular recognition event must occur between pre- and post-synaptic cells (Sperry, 1963). He proposed that this was achieved by expression of complementary chemoaffinity molecules that would serve as molecular cell labels to allow the two cells to recognize each other; he called these "individual identification tags."

Since Sperry, a large number of neural cell adhesion proteins have been identified that are localized to synapses, are required for proper synapse formation or stability, and in some cases have synaptogenic activity. These proteins are good candidates for fulfilling the role of Sperry determinants (Chia et al., 2013; Colon-Ramos, 2009; Hong and Luo, 2014; Langenhan et al., 2013; Shapiro et al., 2007; Shen and Scheiffele, 2010; Südhof, 2012; Takahashi and Craig, 2013; Williams et al., 2010; Zipursky and Sanes, 2010). But it has not been possible to determine in a definitive manner their possible general role as a code for connectivity, as "individual identification tags," because in most systems where they have been studied there is no complete map of connections.

The picture of the $C$. elegans nervous system that emerges when we consider the posterior nervous system of the $C$. elegans male differs significantly from that of the hermaphrodite. In this region the structures of the neurons and their connections are more typical. Here the complex connectivity would seem to require some type of Sperry-determinant-like functions forming a general molecular code for connectivity.

In the male, the complement of neurons and muscles is augmented by the postembryonic addition of 83 new neurons and 40 new muscles. Altogether 144 neurons and 64 muscles in the tail form a neural network created by over 8,000 separate chemical and gap junction synapses resulting in some 3,200 connected cell pairs (Jarrell et al., 2012). For comparison, the entire hermaphrodite nervous system has 302 neurons and about 4,920 connected cell pairs (not counting muscles) (Varshney et al., 2011; White et al., 1986; this laboratory, unpublished). Unlike in the hermaphrodite, where the en passant synapses are widely dispersed around the nerve ring, in several ganglia, and along the ventral nerve cord, in the male tail most of the synapses are concentrated within a single ganglion, the preanal ganglion (Figure 2). Within this ganglion, neurons are not arranged in constant bundles. 
They branch and intermingle within the 3D volume like strands of spaghetti. In an EM cross section, as many as 200 neurite profiles may be present and an image looks very much like one of mouse brain.

Three of the key structural features of the hermaphrodite nervous system that seemed to simplify the problem of synaptic specificity do not apply to the male mating circuits. First, the neurons that are generated postembryonically are not necessarily born near where they are needed. While some are born within the pre-anal ganglion, others are born and reside in ganglia some distance away, from which they will send out their developing processes into the pre-anal ganglion (Figure 2). Moreover, some of these neurons lie at positions that vary from animal to animal, having been swept into their ganglion by morphogenetic remodeling of the tail region (Sulston et al., 1980). To make their connections, as with neurogenesis in other nervous systems, they send out growth cones that navigate along pathways to reach their targets in the pre-anal ganglion (Jia and Emmons, 2006; Sulston et al., 1980) (Figure 2). The cell lineage would not seem to play a role here in setting initial cell contacts.

Second, many neurons in the mating circuits are branchy. For those navigating to the target area, upon arriving at the pre-anal ganglion, which they enter at various points, their processes bifurcate repeatedly and grow among the other processes as they seek their targets. Several embryonic neurons present in both sexes also grow out new branches postembryonically within the ganglion.

Third, neurites are not constrained to a small number of fixed neighborhoods. Neurite outgrowth and branching make it possible in principle for any neuron to come into contact with any other neuron or muscle. In a preliminary study, the frequency was determined with which the neighbors encountered by one large male-specific interneuron in the pre-anal ganglion, EF3, change along its length. To make a quantitative assessment of the stability of its neighborhoods, cells in contact with EF3 at the ends of 26 segments defined by branch points and endpoints were determined. Fifty-four percent of the 56 neurites in contact at one end of a segment were not present at the other. Thus EF3, rather than running in a constant bundle, experiences a continually changing set of cell contacts. This might reflect active targeting of this cell by the presynaptic neurons. Altogether, 36 different neurons are presynaptic to EF3 over a total of 586 EM sections.

\section{A wealth of detail and complexity}

PC-based annotation of the electron micrographs of the male tail neuropil made it possible to capture more information about the structure than was previously possible. Most notably, the sizes of the individual synapses were measured. Synapse sizes, along with the branchyness of the neurons and the large number of both chemical and gap junction connections each neuron forms with multiple partners present a reproducible structure with a level of complexity that challenges our comprehension. To make some sense of the network, the methods of mathematical graph theory proved to be helpful (Jarrell et al., 2012).

Several features need to be considered. The first is that each neuron in the network interacts with many other neurons and sometimes also muscles. The number of postsynaptic partners 
of a given neuron ranges continuously from just a few to over 40, with a median of around 10; the number of input and gap junction partners have similar distributions. (These distributions are known as the degree distributions of the connectivity graphs in the language of graph theory.) Each type of neuron has a distinct set of pre-, post-, and gap junction synaptic partners. Each of these sets partially overlaps with those of other cell types. How many different such sets are there? Based on the patterns of connectivity as well as other properties (like lineal origin and neurotransmitter expression), the 144 neurons in the network can be assigned to 73 different classes. (This calculation assumes left/right homologs are equivalent cells of a single class and additionally that 73 cells that are not left/ right homologs are of 16 types (Jarrell et al., 2012)). Seventy-three different classes means there are 73 different ways neurons can select targets among the available neurons and muscles.

Once a neuron recognizes an appropriate partner, it must know how to respond. Some pairs of neurons interact exclusively by chemical synapse, some by gap junction synapse, and some by both. Chemical connections are sometimes only in one direction, but a large fraction are reciprocal (albeit usually of different strengths). The prevalence of reciprocal connectivity suggests the possibility that some cell interactions are homophillic.

Inclusion of synapse size in the analysis revealed that the interaction between pre- and postsynaptic cells results in a specified strength of connection (the so-called edge weight in the connectivity graph). Jarrell et al. (2012) drew this important conclusion by examining the similarity of connectivity of equivalent neurons. Based on the morphological sizes and numbers of synaptic contacts, each neuron has both strong and weak partners, differing up to 100 -fold in connection strength. It was found by comparing the sets of connections made by equivalent neurons that both the strong and weak sets exhibited statistically significant similarity. This observation indicates that not only the set of synaptic partners and the type and direction of each synaptic connection, but also the strength of synaptic interaction is genetically specified for each interacting cell pair.

How do growth cones find their targets in the pre-anal ganglion? Do they follow reproducible pathways adhering to predefined neighbors? While a detailed threedimensional reconstruction has not yet been carried out, comparison of the variable branching architectures of left/right homologs, presumptively equivalent cells, reveals little if any suggestion of such a reproducible architecture. As an example, consider a left/right pair of sensory neurons with dendritic endings in rays, R2BL and R2BR. These neurons are among the ones mentioned above that have cell bodies residing at variable positions in ganglia widely separated from the pre-anal ganglion. Their growth cones enter the preanal ganglion independently on opposite sides from commissures that respectively traverse around the left and right sides of the body. While the neurites invade a similar region of the ganglion, their branching structures bear no similarity and little relationship to synapse formation (Figure 3). Some branches are formed that make no synapses, while others reach a productive region and make many synapses. Most branches grow beyond the last synapse. It looks like a random search. Yet in spite of these differences, these neurons make similar (albeit far from identical) sets of connections (Figure 3). 


\section{Can clues as to the identity of "individual identification tags" be found in the patterns of connections?}

The complexity strongly implies the existence of a relatively large set of cell labels that collectively carry the instructions for forming the network. As mentioned above, elsewhere in the $C$. elegans nervous system extracellular cell labels that induce or inhibit synapse formation can be expressed in adjacent hypodermal or glial cells. However, most neurites in the male preanal ganglion only contact other neurites, so it is likely that the neurites themselves bear cell labels. It seems there must be many such labels in order to generate the 73 distinct subsets of synaptic connections. White et al (1986) similarly pointed out that even in the hermaphrodite nervous system the code must be combinatorial since the different sets of postsynaptic cells intersect. Marshalling an argument based on parsimony, can evidence for such overlapping sets of labels be deduced from the patterns of connections?

As an example, consider how the 36 cells that target EF3, which are of 21 different types, recognize it. There could be 21 different labels expressed by EF3, one for each pre-synaptic cell type. More parsimoniously, EF3 could express a single label (or a set of labels) that is recognized by each of the pre-synaptic cells. In this case, the members of the set of presynaptic cells should all express the same receptor(s) that recognizes the EF3 label(s). Moreover, the very likely identical EF1 and EF2 neurons, which have similar sets of presynaptic partners, should express the same label(s) as EF3. A fruitful experimental approach could be to search for potential extracellular or transmembrane label proteins whose expression patterns match these respective sets of pre- and post-synaptic cells.

As the classes of neurons presynaptic to EF1, EF2, and EF3 are also presynaptic to additional but in each case different postsynaptic cells, in addition to sharing recognition for the EF determinant, they must express additional recognition molecules. In a combinatorial code for synapse formation, each neuron might express a number of these determinants. The number that matched those expressed by another cell might determine the probability of synapse formation. It is in this manner that the number of matching isomorphs of DSCAM proteins expressed by neurons in Drosophila determines the probability of repulsion between them (Hattori et al., 2008). Similarly in mammals, combinations of protocadherin isoforms determine single-cell identities (Thu et al., 2014). Multiple determinants, each contributing to a probability of synapse formation, would provide a flexibility that could allow for gradual changes in synaptic strength and ultimately evolution of connectivity.

In a second example, consider the pre-anal ganglion interneuron/motorneuron pair PVV and PDB. Male-specific, post-embryonic PVV targets ventral bodywall motor circuitry. Sex nonspecific, embryonic PDB innervates dorsal bodywall motorneurons and muscles. Both neurons are extensively branched (the PDB branches are male-specific) and receive significant input, particularly sensory input (Figure 4). PVV and PDB are clearly not equivalent cells. Yet they receive very similar patterns of synaptic inputs (Figure 4). Among the 18 ray neuron types, four have no input to either while nine have input to both. Only five have input to just one or the other, and among these, only R5B has relatively strong input to just one, PVV. Once again, a parsimonious explanation is that, with regard to recognition for synapse formation, as far as many neurons are concerned PVV and PDB are 
indistinguishable. A subset of ray and other neurons have receptors that recognize and interact with the PVV and PDB labels more or less avidly while all other cells do not. A search could be made for genes whose pattern of expression could explain this connectivity.

\section{Candidates for cell labels}

As mentioned above, studies in many systems have identified cell surface recognition and adhesion proteins expressed at synapses. These variously are necessary for normal synaptogenesis or synapse function or maintenance, and many can induce synapse formation in cell culture experiments. The $C$. elegans genome contains numerous homologs of these genes, as well as genes encoding similar transmembrane proteins with recognizable proteinprotein interaction extracellular domains. Hobert has provided a valuable listing (Hobert, 2013). Thus there is no dearth of candidate proteins for subserving the function of Sperry's tags. White and coworkers, nearly 30 years ago, looked forward to the identification of synaptic recognition determinants or critical cell adhesion molecules through analysis of $C$. elegans behavioral mutants (White, 1985; White et al., 1986). It hasn't happened and one wonders why. The system must be so over-determined that it is not readily penetrable through forward genetic screens. This should perhaps not be surprising. The wiring is so complex it cannot be constructed exactly the same every time. If that were necessary, developmental error would preclude a functional outcome. We now have direct evidence that some of the genes very likely to be involved (a prominent example is neurexin, which is widely-expressed in the $C$. elegans nervous system), have no obvious loss-of-function mutant phenotype. This state of affairs is likely due to redundancy at two levels - each synaptic connection is probably determined by multiple interactions and, secondly, function of the network is robust to changes in the strengths of individual connections.

\section{Conclusion}

The availability of a complete connectome allows us to view the problem of synaptic specificity both from a global and a detailed perspective. The detailed information makes the wiring problem seem more difficult but the global view provides a way to solve it. By examination of the whole, including in the analysis both chemical synapses and gap junction connections, as opposed to one synaptic connection at a time, and comparing the entire set of connections to the expression patterns of the neural cell adhesion proteins that are the presumptive cell labels for synapse formation, it should be possible to discern a pattern that reveals the underlying code. The landscape of cell labels is expected to act progressively over a series of steps - to guide growth cones to a general target area, to promote or inhibit contact with other neurons, to adhere partners together, and finally to trigger synapse formation, or possibly to inhibit it - each step making an incremental contribution to specificity. The expression patterns of the cell labels that encode the wiring must map onto the pattern of synaptic connections in a logical way. The wiring diagram is encoded in the protein-protein interaction domains of the cell labels, and in the genomic regulatory sequences that govern their cell-specific expression. 


\section{Acknowledgments}

Work in the author's laboratory has been supported by grants from the US National Institutes of Health and the The G. Harold and Leila Y. Mathers Charitable Foundation.

\section{References}

Brenner S. The genetics of Caenorhabditis elegans. Genetics. 1974; 77:71-94. [PubMed: 4366476]

Brenner S. In the Beginning Was the Worm .... Genetics. 2009; 182:413-415. [PubMed: 19506024]

Chalfie M, Sulston JE, White JG, Southgate E, Thomson JN, Brenner S. The neural circuit for touch sensitivity in Caenorhabditis elegans. The Journal of Neuroscience. 1985; 5:956-964. [PubMed: 3981252]

Chia PH, Li P, Shen K. Cellular and molecular mechanisms underlying presynapse formation. The Journal of Cell Biology. 2013; 203:11-22. [PubMed: 24127213]

Colon-Ramos, DA. Current Topics in Developmental Biology. Academic Press; 2009. Chapter 2 Synapse Formation in Developing Neural Circuits; p. 53-79.

Colón-Ramos DA, Margeta MA, Shen K. Glia Promote Local Synaptogenesis Through UNC-6 (Netrin) Signaling in C. elegans. Science. 2007; 318:103-106. [PubMed: 17916735]

Ding M, Chao D, Wang G, Shen K. Spatial regulation of an E3 ubiquitin ligase directs selective synapse elimination. Science. 2007; 317:947-951. [PubMed: 17626846]

Durbin, RM. Studies on the development and organization of the nervous system of Caenorhabditis elegans. Cambridge, UK: Cambridge University; 1987.

Emmons SW. The beginning of connectomics: a commentary on White et al. (1986) 'The structure of the nervous system of the nematode Caenorhabditis elegans'. Phil Trans R Soc Lond B. 2015; 370

Hattori D, Millard SS, Wojtowicz WM, Zipursky SL. Dscam-mediated cell recognition regulates neural circuit formation. Annu Rev Cell Dev Biol. 2008; 24:597-620. [PubMed: 18837673]

Hobert, O. The neuronal genome of Caenorhabditis elegans. WormBook, T.C.e.R. Community., editor. 2013.

Hong W, Luo L. Genetic Control of Wiring Specificity in the Fly Olfactory System. Genetics. 2014; 196:17-29. [PubMed: 24395823]

Howell K, White JG, Hobert O. Spatiotemporal control of a novel synaptic organizer molecule. Nature. 2015 advance online publication.

Jarrell TA, Wang Y, Bloniarz AE, Brittin CA, Xu M, Thomson JN, Albertson DG, Hall DH, Emmons SW. The connectome of a decision-making neural network. Science. 2012; 337:437-444. [PubMed: 22837521]

Jia L, Emmons SW. Genes that control ray sensory neuron axon development in the Caenorhabditis elegans male. Genetics. 2006; 173:1241-1258. [PubMed: 16624900]

Kasthuri N, Hayworth Kenneth J, Berger Daniel R, Schalek Richard L, Conchello José A, KnowlesBarley S, Lee D, Vázquez-Reina A, Kaynig V, Jones Thouis R, et al. Saturated Reconstruction of a Volume of Neocortex. Cell. 162:648-661.

Klassen MP, Shen K. Wnt Signaling Positions Neuromuscular Connectivity by Inhibiting Synapse Formation in C. elegans. Cell. 2007; 130:704-716. [PubMed: 17719547]

Langenhan T, Aust G, Hamann J. Sticky Signaling-Adhesion Class G Protein-Coupled Receptors Take the Stage. Sci Signal. 2013; 6:re3. [PubMed: 23695165]

Poon VY, Klassen MP, Shen K. UNC-6/netrin and its receptor UNC-5 locally exclude presynaptic components from dendrites. Nature. 2008; 455:669-673. [PubMed: 18776887]

Shao Z, Watanabe S, Christensen R, Jorgensen Erik M, Colón-Ramos Daniel A. Synapse Location during Growth Depends on Glia Location. Cell. 2013; 154:337-350. [PubMed: 23870123]

Shapiro L, Love J, Colman DR. Adhesion Molecules in the Nervous System: Structural Insights into Function and Diversity. Annual Review of Neuroscience. 2007; 30:451-474.

Shen K, Bargmann CI. The immunoglobulin superfamily protein SYG-1 determines the location of specific synapses in C. elegans. Cell. 2003; 112:619-630. [PubMed: 12628183] 
Shen K, Scheiffele P. Genetics and cell biology of building specific synaptic connectivity. Annual Review of Neuroscience. 2010; 33:473-507.

Sperry RW. Chemoaffinity in the orderly growth of nerve fiber patterns and connections. Proc Natl Acad Sci U S A. 1963; 50:703-710. [PubMed: 14077501]

Südhof, Thomas C. The Presynaptic Active Zone. Neuron. 2012; 75:11-25. [PubMed: 22794257]

Sulston JE. Neuronal Cell Lineages in the Nematode Caenorhabditis elegans. Cold Spring Harbor Symposia on Quantitative Biology. 1983; 48:443-452.

Sulston JE, Albertson DG, Thomson JN. The Caenorhabditis elegans male: postembryonic development of nongonadal structures. Dev Biol. 1980; 78:542-576. [PubMed: 7409314]

Sulston JE, Schierenberg E, White JG, Thomson JN. The embryonic cell lineage of the nematode Caenorhabditis elegans. Developmental Biology. 1983; 100:64-119. [PubMed: 6684600]

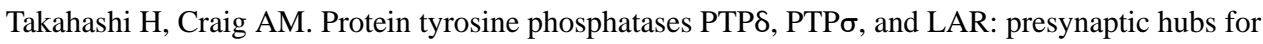
synapse organization. Trends in Neurosciences. 2013; 36:522-534. [PubMed: 23835198]

Thu, Chan A., Chen, Weisheng V., Rubinstein, R., Chevee, M., Wolcott, Holly N., Felsovalyi, Klara O., Tapia, Juan C., Shapiro, L., Honig, B., Maniatis, T. Single-Cell Identity Generated by Combinatorial Homophilic Interactions between $\alpha, \beta$, and $\gamma$ Protocadherins. Cell. 2014; 158:1045-1059. [PubMed: 25171406]

Varshney LR, Chen BL, Paniagua E, Hall DH, Chklovskii DB. Structural properties of the Caenorhabditis elegans neuronal network. PLoS Computational Biology. 2011; 7:e1001066. [PubMed: 21304930]

White JG. Neuronal connectivity in Caenorhabditis elegans. Trends in Neuroscience. 1985; 8:277-283.

White JG, Southgate E, Thomson JN, Brenner S. Factors that determine connectivity in the nervous system of Caenorhabditis elegans. Cold Spring Harb Symp Quant Biol. 1983; 48(Pt 2):633-640. [PubMed: 6586380]

White JG, Southgate E, Thomson JN, Brenner S. The structure of the nervous system of the nematode Caenorhabditis elegans. Philosophical Transactions of the Royal Society, Series B Biological Sciences. 1986; 314:1-340.

Williams ME, de Wit J, Ghosh A. Molecular Mechanisms of Synaptic Specificity in Developing Neural Circuits. Neuron. 2010; 68:9-18. [PubMed: 20920787]

Zipursky SL, Sanes JR. Chemoaffinity revisited: Dscams, protocadherins, and neural circuit assembly. Cell. 2010; 143:343-353. [PubMed: 21029858] 


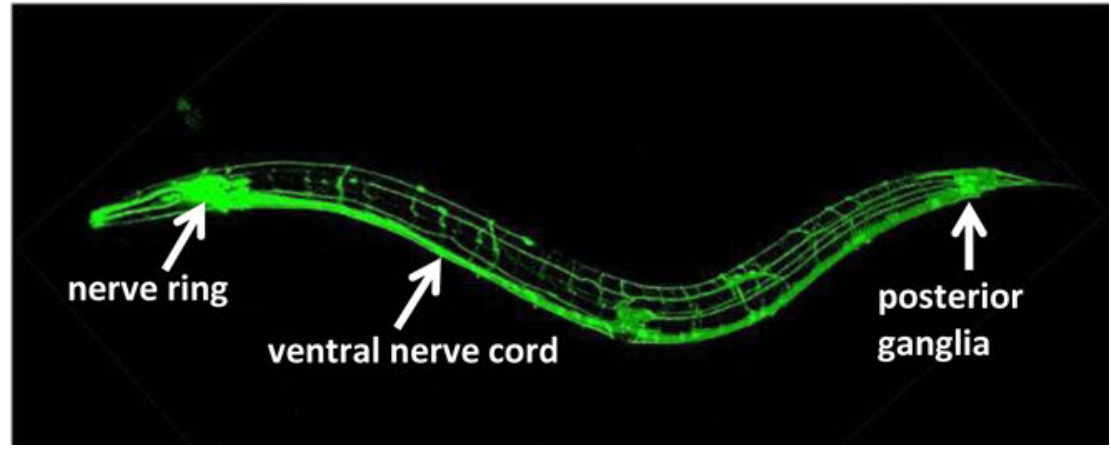

Figure 1.

The nerve bundles of the $C$. elegans nervous system, visualized by a reporter gene that expresses the fluorescent protein GFP in all of the neurons. (Image from Hang Ung, JeanLouis Bessereau laboratory, France.) 


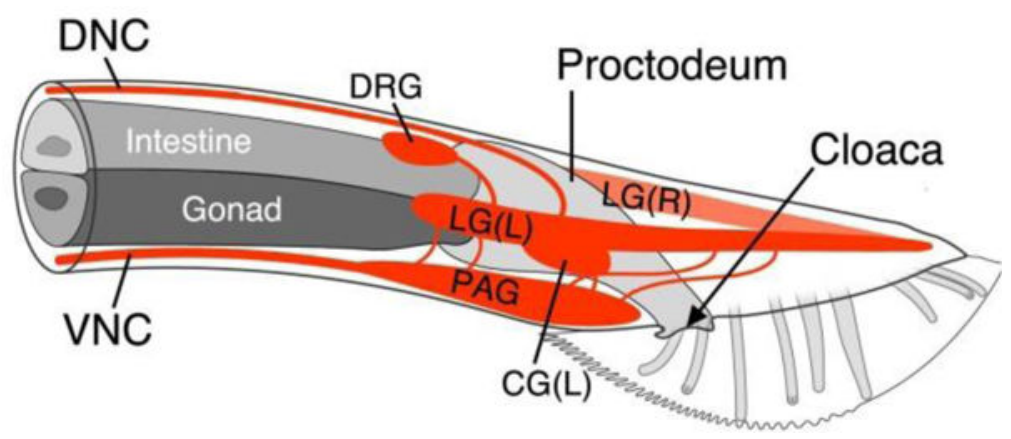

Figure 2.

Ganglia in the $C$. elegans male tail. CG(L), left cloacal ganglion; DNC, dorsal nerve cord; DRG, dorsorectal ganglion; LG(L), left lumbar ganglion; LG(R), right lumbar ganglion; PAG, preanal ganglion; VNC, ventral nerve cord; right cloacal ganglion not shown. Cell bodies are distributed in each of the six ganglia. Synapses can be found throughout, but are situated primarily within the PAG. Neurons outside this ganglion send processes through commissures into it. (Image from WormAtlas, http://www.wormatlas.org.) 

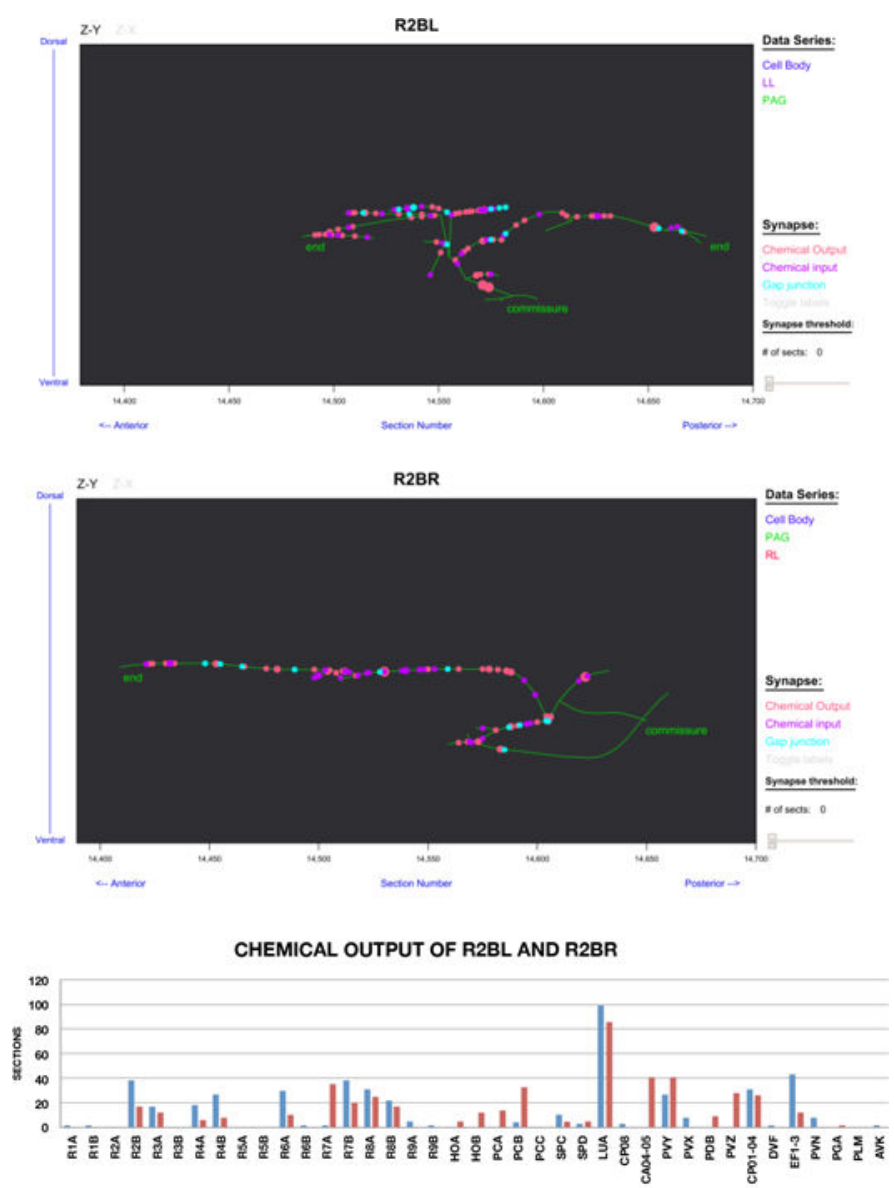

CHEMICAL INPUT TO R2BL AND R2BR

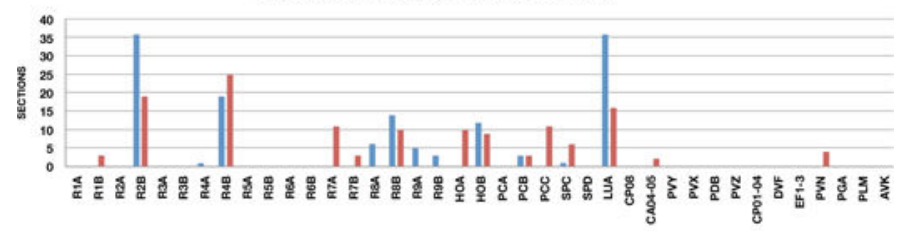

GAP JUNCTIONS OF R2BL AND R2BR

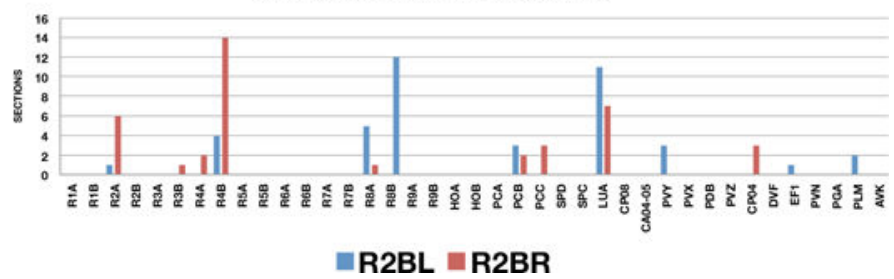

Figure 3.

Homologous neurons have dissimilar branching structures in the preanal ganglion but similar sets of synaptic partners. Upper panels: the processes and synapses in the PAG of a pair of homologous neurons, the ray sensory neurons R2BL and R2BR. The cell bodies of these neurons are respectively in the left and right lumbar ganglia (Figure 2). Pink circles show sites of chemical synaptic output, magenta circles are chemical input, and blue circles are gap junctions. These maps are from Wormwiring.org. Lower panels: histograms comparing the synaptic relationships made by R2BL and R2BR. The neurons listed along 
the bottom are the 68 neurons (out of 170) with synaptic interaction of some kind with one or both of these two neurons Data for left/right homologs of R2BL and R2BR partners are combined; e.g. R1A represents the interaction with $\mathrm{R} 1 \mathrm{AL}$ plus $\mathrm{R} 1 \mathrm{AR}$. The vertical axis gives the total morphological strength of interaction in number of EM serial sections. Note the differences in scales. 

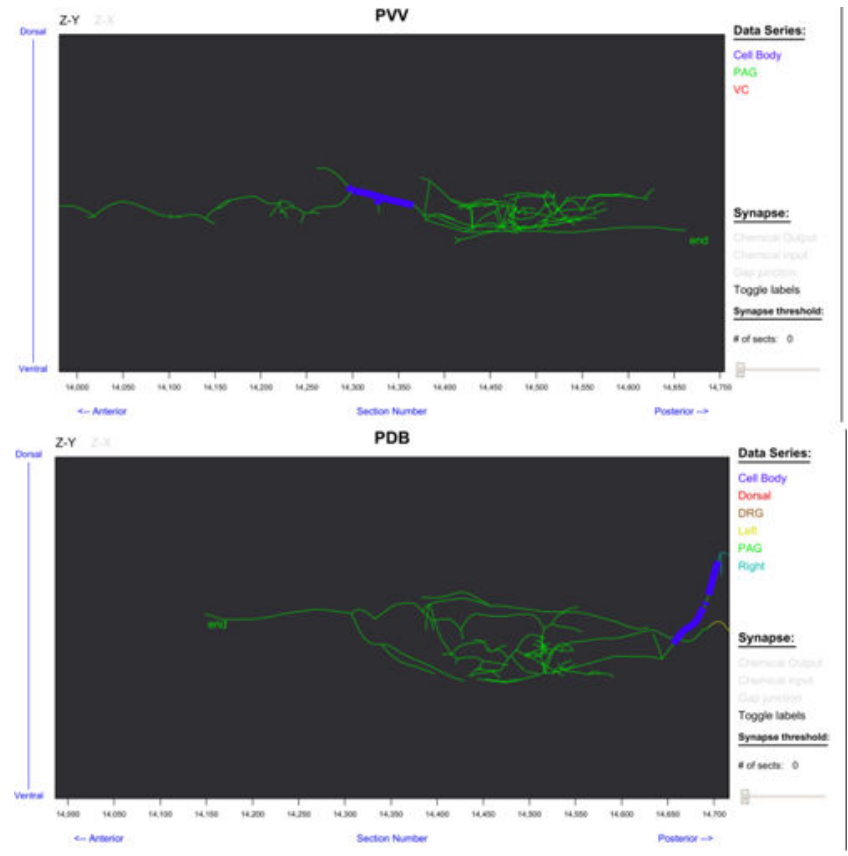

RAY CHEMICAL INPUT TO PVV (BLUE) AND PDB (RED)

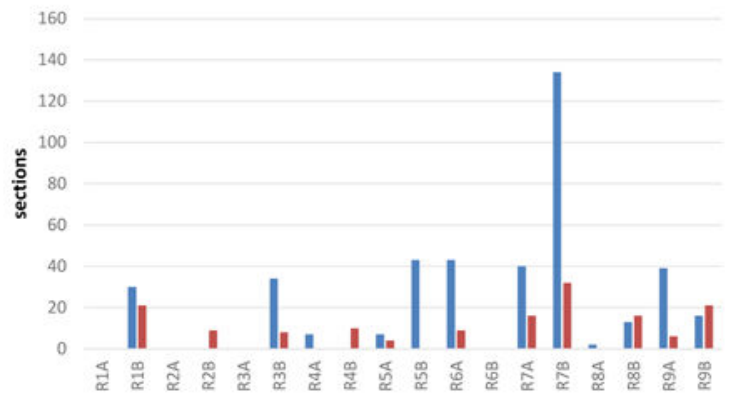

NON-RAY CHEMICAL INPUT TO PVV (BLUE) AND PDB (RED)

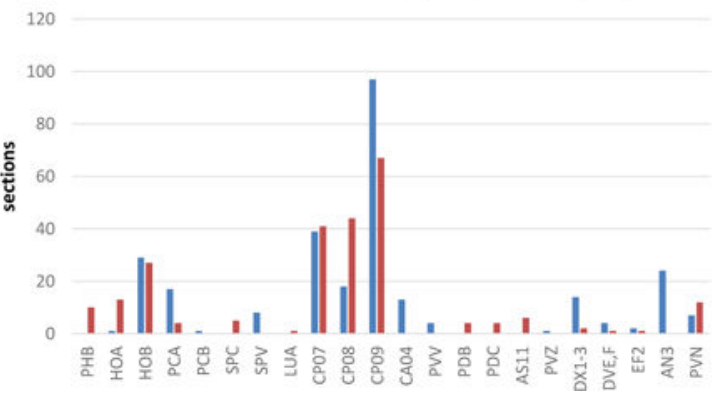

Figure 4.

PVV and PDB are distinct neurons with similar patterns of synaptic input. Top panels show the overlapping branching structures of PVV and PDB in the preanal ganglion. PVV extends further anteriorly into the ventral cord, while PDB sends a process posteriorly around the end of the tail to the dorsal side. Both have output onto bodywall muscles. The blue boxes are the cell bodies. The horizontal axes show the serial section number of the transverse serial sections (A-P axis); the vertical axis shows position along the D-V axis; the maps are flattened along the L-R axis. The top histogram shows connectivity data for all the ray 
neurons; R1A(L/R), R2A(L/R), R3A(L/R) and R6B(L/R) make no connection to either PVV or PDB. The bottom histogram shows data for all the remaining neurons that make a chemical connection to either neuron. Data for left/right homologs and other equivalent neurons are combined. 